\title{
Was ist kognitives Altern? \\ Begriffsbestimmung und Forschungstrends
}

ULMAN LINDENBERGER

Was ist Alter(n)?, Ursula M. Staudinger, Heinz Häfner (Hg.),

Schriften der Mathematisch-naturwissenschaftlichen Klasse

der Heidelberger Akademie der Wissenschaften, Nr. 18 (2008), Springer Verlag Berlin Heidelberg,

S. $69-82$ 



\title{
Was ist kognitives Altern? \\ Begriffsbestimmung und Forschungstrends
}

\author{
Ulman Lindenberger
}

\section{Einleitung*}

Das Alter kann von den Lebensperioden, die ihm vorausgehen und nachfolgen, nicht klar abgegrenzt werden. Wir können es nur als Veränderung, als Altern, erforschen und gestalten. Die Vielfältigkeit, Interdependenz und Dynamik der biologischen und kulturellen Einflusssysteme, die das Altern bestimmen, stellen auch die kognitive Alternsforschung vor große methodologische Herausforderungen, also jenen Bereich, der sich mit altersbezogenen Veränderungen im Wahrnehmen, Denken und Handeln befasst. So können kognitive Leistungsunterschiede zwischen Personen, die zu einem gegebenen historischen Zeitpunkt unterschiedlich alt sind, neben, anstatt von oder in Interaktion mit primär biologisch bedingten Reifungs- oder Alterungsprozessen auch historische Veränderungen in leistungsrelevanten Umweltbedingungen zum Ausdruck bringen. Weiterhin können Leistungssteigerungen, die bei der wiederholten Messung kognitiver Fähigkeiten in Längsschnittuntersuchungen auftreten, Lerneffekte durch wiederholte Aufgabenbearbeitung erfassen, die mit Reifungs- oder Alterungsprozessen interagieren und die deren Bestimmung erschweren. Schließlich gestaltet sich im hohen Alter die Trennung zwischen ,normalen“Alterungsprozessen, alterskorrelierten Pathologien sowie sterbebezogenen Veränderungen als schwierig.

Diese methodologischen Komplikationen liegen in der Natur der Sache, sie verweisen auf die heterogene und dynamische Qualität menschlicher Entwicklungsprozesse. Deswegen entfaltet und präzisiert die Arbeit an methodologischen Problemen der Alternsforschung zugleich deren Gegenstand sowie die Konzepte und Annahmen, die dessen Erforschung zugrunde liegen. So haben Entwicklungspsychologen seit jeher darauf hingewiesen, dass dem Alter oder der Lebenszeit für sich genommen kein erklärender Gehalt zukommt (z. B. Wohlwill, 1970). Vielmehr fungiert Alter als Träger zahlreicher sowie miteinander interagierender biologischer und kultureller Einflüsse. Dementsprechend kann es in der Entwicklungspsychologie auch kein einheitliches methodisches Vorgehen geben. Erforderlich ist vielmehr die gezielte, der jeweiligen

\footnotetext{
* Der Verfasser dankt Paul Baltes, Yvonne Brehmer, Shu-Chen Li, Martin Lövdén, Florian Schmiedek und Ursula Staudinger für hilfreiche Gespräche über die Inhalte dieses Beitrags. Er dankt außerdem den Mitgliedern der Leopoldina-acatech-Arbeitsgruppe „Chancen und Probleme einer alternden Gesellschaft: Die Welt der Arbeit und des lebenslangen Lernens " für anregende Diskussionen. Teile des vorliegenden Beitrags basieren auf früheren Veröffentlichungen (Brehmer \& Lindenberger, 2008; Lindenberger, 2007a; Lindenberger, Li, Lövdén \& Schmiedek, 2007; Schmiedek \& Lindenberger, 2007).
} 
Fragestellung und den jeweiligen Hypothesen angepasste Kombination verschiedener Strategien, um zu gehaltvollen und ausreichend abgesicherten inhaltlichen Interpretationen von Forschungsergebnissen zu gelangen. Diese Interpretationen müssen die Reflektion über das Ausmaß und die Dimensionen der Generalisierbarkeit der Befunde, das heißt umgekehrt ihre Bedingtheit durch den entwicklungsgeschichtlichen Kontext, von vornherein einschließen (Schmiedek \& Lindenberger, 2007).

Vor dem Hintergrund dieser methodologischen Kautelen werde ich im Folgenden das Thema dieses Bandes, ,Was ist Alter? “, kognitionspsychologisch bearbeiten. Ich werde zunächst den Gegenstand der kognitiven Alternsforschung allgemein bestimmen und anschließend ausgewählte aktuelle Trends der aktuellen Forschung näher darstellen.

\section{Kognitives Altern: Zur Bestimmung eines Forschungsgegenstands}

Personen stehen durch ihr Verhalten in Wechselwirkung mit ihrer sozialen und physischen Umwelt (siehe Abb. 1; vgl. Lindenberger, Li \& Bäckman, 2006). Einerseits

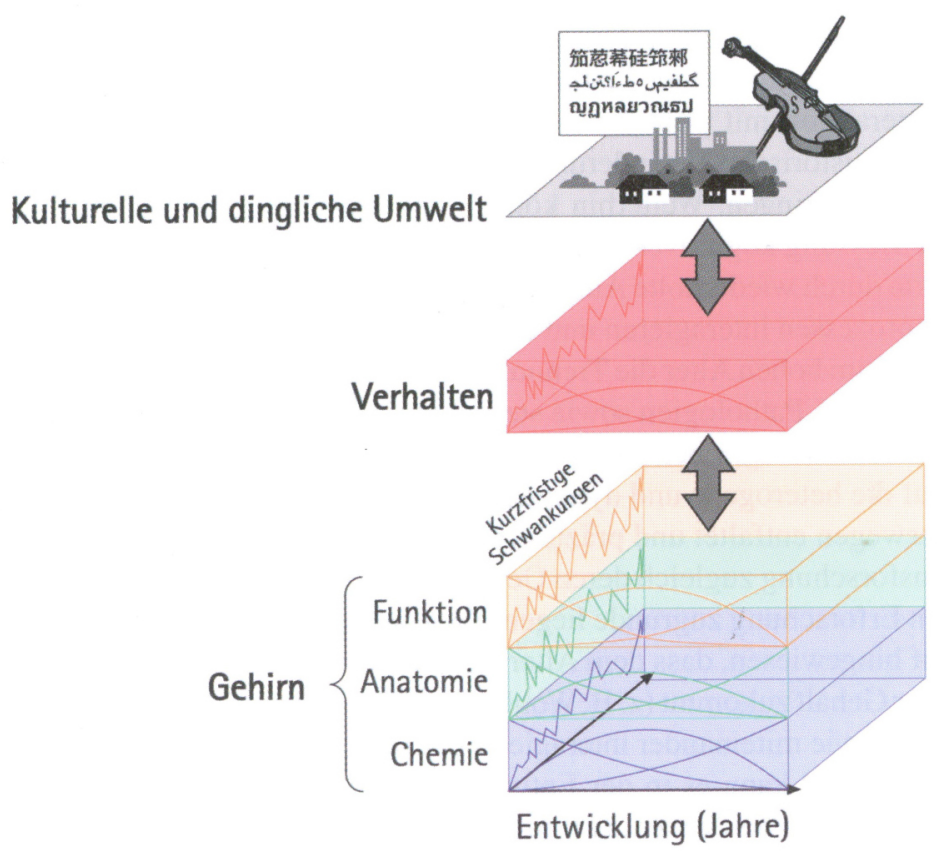

Abb. 1. Umwelt und Gehirn als Ursachen und Wirkungen kurzfristiger und langfristiger Veränderungen in Verhaltensmustern.

Veränderungen der Beziehungen zwischen Gehirn und Verhalten reflektieren Wechselwirkungen zwischen Reifung, Lernen und Seneszenz. Die Bestimmung zentraler Mechanismen dieser Wechselwirkungen erfordert Theorien und empirische Methoden zur Integration der Befunde über Inhaltsbereiche, Zeitskalen und Analyseebenen. Nach Lindenberger, Li und Bäckman (2006). 
bestimmen sowohl die physikalische und kulturelle Umwelt als auch das Gehirn die Verhaltensentwicklung. Andererseits verändern Individuen durch ihr Verhalten sowohl ihre Umwelt als auch ihr Gehirn. Umwelt und Gehirn sind demnach Ursachen, aber auch Folgen kurz- und langfristiger Veränderungen in Verhaltensmustern. Die Komponenten dieses Systems - Umwelt / Verhalten / Gehirn - sind gekoppelt, nicht aufeinander reduzierbar und bestimmen durch rekursive Selbstregulation die Entwicklung der Person.

Wie die Entwicklungspsychologie insgesamt verfolgt auch die kognitive Alternsforschung das Ziel, Invarianz und Variabilität, Stabilität und Veränderung von Verhaltensrepertoires im Lebensverlauf zu erklären (Molenaar, in Druck; Nesselroade, Gerstorf, Hardy \& Ram, 2007). Im eigentlichen Sinne entwicklungspsychologisch sind die Erklärungen dann, wenn sie Mechanismen und Organisationsformen anführen, die innerhalb der Person wirksam sind. So rufen die Frühverrentung oder ein schöner Frühlingstag psychische Wirkungen hervor (die sich von Person zu Person unterscheiden können), stellen aber selbst keine psychologischen Mechanismen oder Erklärungen dar.

Zur näheren Bestimmung altersbezogener Verhaltensänderungen ist es sinnvoll, zwischen drei Klassen von Mechanismen zu unterscheiden: Reifung, Lernen und Seneszenz. Reifung und Seneszenz erstrecken sich über die gesamte Lebensspanne, sind jedoch in der frühen beziehungsweise späten Ontogenese in besonders starkem Maße wirksam. Lernen bezieht sich auf Veränderungen in der Folge von Interaktionen zwischen Verhalten und Umwelt. Es ist offensichtlich, dass Reifung nicht ohne Lernen und Lernen nicht ohne Reifung stattfinden können. Auch hängt die Art und Weise, in der sich Einflüsse der Seneszenz im Verhalten bemerkbar machen, von der gegenwärtigen und vergangenen Lern- und Reifungsbiographie ab. Schließlich beschränkt sich die Wirkung von Reifungsprozessen nicht auf die Kindheit, und Anzeichen von Seneszenz lassen sich nicht auf das hohe Alter begrenzen. So sind Synaptogenese und Neurogenese bis ins hohe Alter nachweisbar (Kempermann, 2005; vgl. auch Kempermann in diesem Band), und das Nachlassen der dopaminergen Neuromodulation, das zu den wichtigsten Merkmalen der kognitiven Alterung gehört, beginnt bereits im frühen Erwachsenenalter (Bäckman, Nyberg, Lindenberger, Li \& Farde, 2006).

Es wäre also abwegig, Reifung mit Kindheit und Seneszenz mit Alter gleichzusetzen. Vielmehr bereichern und begrenzen Reifung, Seneszenz und Lernen einander über die gesamte Lebensspanne und sind als interagierende Kräfte im GehirnVerhalten-Umwelt-System wirksam. Beim Versuch, diese Interaktionen zu verstehen, nehmen Verhaltenwissenschaftler im allgemeinen und Entwicklungspsychologen im besonderen eine zentrale Stellung ein, da sie über ein reichhaltiges Repertoire an Methoden und experimentellen Zugängen verfügen, die Veränderungen in der Organisation des Verhaltens beschreiben.

Dabei steht die kognitive Alternsforschung aus Sicht der Psychologie der Lebensspanne (vgl. Baltes, Lindenberger \& Staudinger, 2006) vor drei konzeptuellen und empirischen Integrationsleistungen (Lindenberger et al., 2007):

Erstens kommt es darauf an, verschiedene Funktionsbereiche und Aspekte des Verhaltens wie Kognition und Sensomotorik oder Kognition, Affekt und Motivation 
von vornherein aufeinander zu beziehen und nicht erst im Nachhinein durch Zusatzannahmen miteinander zu verknüpfen. So hängen, wie weiter unten noch im Einzelnen gezeigt wird, sensomotorische und kognitive Funktionsbereiche im hohen Alter enger miteinander zusammen als im mittleren Erwachsenenalter, so dass Altersveränderungen in beiden Bereichen besser verstanden werden können, wenn man deren Wechselwirkungen von vornherein berücksichtigt (Schaefer, Huxhold \& Lindenberger, 2006). Ähnliches gilt für die Entwicklung von Kognition und sozialer Interaktion sowie für die Entwicklung von Emotion und Motivation.

Zweitens bedarf es Theorien und Methoden, welche die für Veränderungen relevanten Mechanismen miteinander in Beziehung setzen und zwar besonders hinsichtlich unterschiedlicher Zeitskalen (Li, Huxhold \& Schmiedek, 2004). Kurzfristige Schwankungen im Verhalten, die sich auf momentane Verhaltensäußerungen (Items), Tage oder Wochen beziehen, sind eher vorübergehend und reversibel, Veränderungen, die sich auf Monate, Jahre oder Jahrzehnte beziehen, eher kumulativ und kaum rückgängig zu machen. Das Auffinden von Verbindungen zwischen Veränderungen auf unterschiedlichen Zeitskalen ist von heuristischem Wert, weil sich Mechanismen über kürzere Zeiträume in der Regel besser beobachten lassen als über längere Zeiträume. So postuliert eine Reihe von Theorien des kognitiven Alterns, dass dysfunktionale Schwankungen im Verhalten und in der neuronalen Signalverarbeitung (z. B. bei der Bearbeitungsgeschwindigkeit einer Wahlreaktionszeitsaufgabe) ein zentrales Kennzeichen seneszenzbedingter Defizite darstellen, bei dem genannten Beispiel in der Zuverlässigkeit der Informationsverarbeitung. Anhand von Daten der Berliner Altersstudie konnte im Einklang mit dieser Hypothese nachgewiesen werden, dass ältere Personen, die bei der Bewältigung von Aufgaben an einem bestimmten Tag stärkere Schwankungen hatten, im Laufe der folgenden Jahre auch einen stärkeren Abbau ihrer kognitiven Leistungen zeigten als Personen mit geringeren Schwankungen (Lövdén, Li, Shing \& Lindenberger, 2007; siehe Abb. 2). ${ }^{1}$

Drittens geht es der kognitiven Alternsforschung darum, neuronale und kognitive Mechanismen altersbezogener Veränderungen miteinander zu verknüpfen. Durch den Einsatz moderner neurowissenschaftlicher Verfahren können die neurofunktionalen, neurochemischen und neuroanatomischen Manifestationen von Alterungsprozessen und ihre Beziehungen zum Verhalten besser bestimmt werden als je zuvor. Die Beziehungen zwischen neuronaler und behavioraler Analyseebene sind zwischen und innerhalb von Personen variabel und verändern sich selbst im Laufe des Lebens (Li, 2003; Li \& Lindenberger, 2002). Altersbezogene Veränderungen im Verhaltensrepertoire gehen folglich mit kontinuierlichen, mehr oder minder reversiblen Veränderungen der Beziehungen zwischen Verhaltens- und Gehirnzuständen einher. Einige dieser Veränderungen sind relativ universell, andere wiederum reflektieren genetische Unterschiede, Besonderheiten der Lerngeschichte sowie die Pfadabhängigkeit von Ent-

\footnotetext{
${ }^{1}$ Dies bedeutet nicht, dass kurzfristige Schwankungen generell dysfunktional und durchweg als frühe Indikatoren biologischer Abbauprozesse anzusehen sind. Zum Beispiel können Schwankungen, wenn sie während der Bearbeitung neuartiger und schwieriger Aufgaben auftreten, die Suche nach geeigneten Strategien anzeigen und späteren Lernerfolg positiv vorhersagen (vgl. Allaire \& Marsiske, 2005; Siegler, 1994).
} 


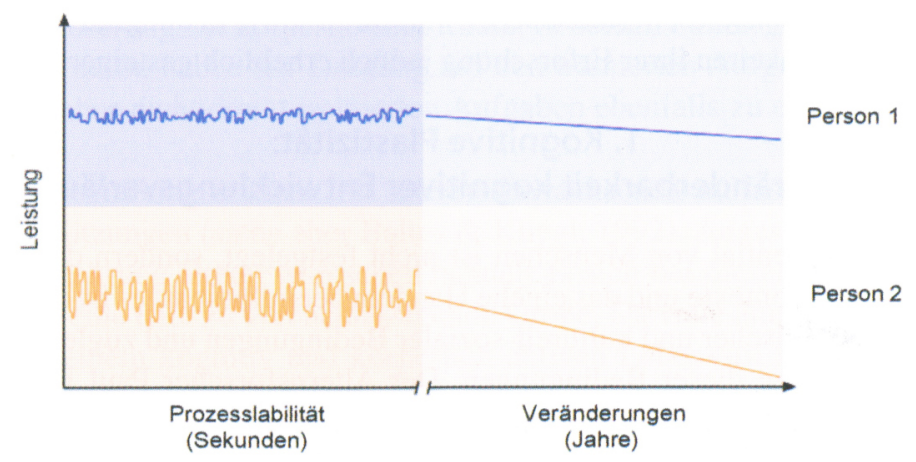

Abb. 2. Beispiel zur Kopplung kurzfristiger Fluktuationen und langfristiger Veränderungen.

Ältere Erwachsene mit größeren momentanen Leistungsschwankungen zeigen über den Zeitraum mehrerer Jahre einen stärkeren Abbau kognitiver Fähigkeiten als ältere Erwachsene mit geringeren Leistungsschwankungen. Die empirischen Befunde stimmen mit dieser Annahme überein (Lövdén et al., 2007; MacDonald, Hultsch \& Dixon, 2003). Modifiziert nach Lindenberger, Li \& Bäckman (2006).

wicklungsprozessen (Molenaar, Boomsma \& Dolan, 1991). Im Ergebnis ergibt sich ein vielschichtiges Bild, das auf der einen Seite die Vielfalt und Formbarkeit von Entwicklungswegen verdeutlicht, auf der anderen Seite aber auch die Grenzen aufzeigt, die Reifung und Alterung, allgemeine Gesetze der neuronalen Organisation sowie kulturelle und physikalische Gesetzmäßigkeiten der Umwelt der menschlichen Entwicklung setzen (Baltes et al., 2006).

Die Forschung zu kognitiven Prozessen im Alter ist gemäß dieser Gegenstandsbestimmung besonders dann erfolgversprechend, wenn sie Inhaltsbereiche, Zeitskalen und Analyseebenen integriert. Bestimmte Forschungsmethoden unterstützen diese Integrationsanforderungen in besonderer Weise. So sind in statistischer Hinsicht sogenannte Mehrebenenmodelle beziehungsweise latente Wachstumsmodelle besonders gut dazu geeignet, mehrdimensionale und zeitlich geschichtete Datensätze zu strukturieren. Dynamische Varianten dieser Modelle (McArdle \& Hamagami, 2001) erlauben die Erkundung gerichteter Veränderungsbeziehungen (siehe z. B. Ghisletta \& Lindenberger, 2003, 2004; Lövdén, Ghisletta \& Lindenberger, 2005).

In konzeptueller Hinsicht sind neuronale Netzwerkmodelle zu einem zentralen Werkzeug der Integration neuronaler und behavioraler Theoriebildung avanciert. Ein prominentes Beispiel ist die von Shu-Chen Li und anderen vorgeschlagene Theorie des kognitiven Alterns über das Nachlassen der dopaminergen Neuromodulation und dessen Folgen (Li \& Lindenberger, 1999; Li, Lindenberger \& Sikström, 2001; Li, von Oertzen \& Lindenberger, 2006).

\section{Ausgewählte Trends der kognitiven Alternsforschung}

Vor dem Hintergrund der vorhergehenden Überlegungen erscheinen einige aktuelle Trends der kognitiven Alternsforschung besonders relevant. Zwar sind Fragestellungen, die diese Trends motivieren, zumeist nicht neu; die Fortschritte in empirischen 
Methoden der life sciences, in statistischen Verfahren sowie in integrativen Konzepten haben die Möglichkeiten ihrer Erforschung jedoch erheblich gesteigert.

\section{Kognitive Plastizität: Die Veränderbarkeit kognitiver Entwicklungsverläufe}

Das kognitive Potential von Menschen ist nicht festgelegt, sondern durch günstige oder ungünstige Kontexte und das eigene Handeln beeinflussbar; es ist das gemeinsame Produkt biologischer und kulturell-sozialer Bedingungen und zugleich ein Motor für die Veränderung dieser Bedingungen. Der Alternsforscher Paul Baltes brachte dies unter dem Stichwort des „,bio-kulturellen Ko-Konstruktivismus“ zum Ausdruck (Baltes, Reuter-Lorenz \& Rösler, 2006). Die kognitive Alternsforschung erkundet mit Trainingsstudien Altersunterschiede in der Plastizität des Verhaltens sowie deren Verbindung zu neuronalen Mechanismen.

Die bisherigen Forschungsergebnisse zur kognitiven Plastizität im Alter ergeben ein gemischtes Bild. Einerseits zeigt sich, mit gewissen Ausnahmen im sehr hohen Alter (vgl. Singer, Lindenberger \& Baltes, 2003), dass geistig gesunde ältere Erwachsene in der Lage sind, ihre Leistungen in instruierten und anschließend frei geübten oder unter Anleitung trainierten Aufgaben zu steigern (z. B. Brehmer, Li, Müller, von Oertzen \& Lindenberger, 2007). Dies dokumentiert den Erhalt der Verhaltensplastizität im Alter. Andererseits fanden sich weder in Trainingsstudien zur abstrakten Problemlösefähigkeit (fluide Intelligenz) noch in Trainingsstudien zum episodischen Gedächtnis Hinweise darauf, dass kognitive Interventionen im Erwachsenenalter eine generelle Verbesserung jener Fähigkeiten bewirken, denen die trainierte Aufgabe zugeordnet ist. Wenn ältere Erwachsene also eine Fertigkeit zum seriellen Einprägen und Erinnern von Wörtern erlernen, so haben sie guten Grund zu der Hoffnung, sich künftig Wörter besser in der richtigen Reihenfolge merken zu können (es sei denn, sie scheuen den kognitiven Mehraufwand, der mit der Anwendung dieser Fertigkeit einhergeht). Sie können sich aber vor dem Hintergrund der gegenwärtigen Befundlage keine großen Hoffnungen darauf machen, dass sich ihre Gedächtnisleistungen in Folge des Erwerbs dieser Fertigkeit allgemein verbessern. So wird es den Probanden vermutlich nach Abschluss des Trainings nicht leichter fallen als früher, neue Namen mit neuen Gesichtern zu verknüpfen oder sich daran zu erinnern, was sie am letzten Donnerstagabend getan haben. ${ }^{2}$

Zugleich geben zwei Forschungszweige Anlass zu der Hoffnung, dass bestimmte, auf kognitive Prozesse bezogene Interventionen über den Erwerb eng umgrenzter Fertigkeiten hinaus mit einer übergreifenden Verbesserung kognitiver Leistungen einhergehen und so den Entwicklungsgang der Mechanik der Kognition positiv verändern könnten. Zu diesen Interventionen gehören das Trainieren von Mehrfachaufgaben

\footnotetext{
${ }^{2}$ Daraus lässt sich nicht ohne weiteres ableiten, dass Interventionen, die sich mit im Alltag kaum nutzbaren Gedächtnistechniken befassen, aus angewandter Perspektive obsolet sind. Vielmehr gibt es empirische Hinweise darauf, dass Trainingsprogramme zu Steigerungen des Erlebens intellektueller Kompetenz führen, die positive Wirkungen auf die subjektive Befindlichkeit und das Erleben des eigenen Handlungspotentials haben (Dittmann-Kohli, Lachman, Kliegl \& Baltes, 1991; vgl. Staudinger \& Pasupathi, 2000).
} 
und die Verbesserung der körperlichen Fitness. In beiden Fällen gibt es Hinweise auf Transfer, das heißt, neben der Leistung auf den trainierten Aufgaben kommt es auf verwandten, aber nicht direkt trainierten Aufgaben ebenfalls zu einer Leistungssteigerung.

Kognitive Trainingsstudien mit älteren Erwachsenen umfassten bislang selten mehr als 10 Sitzungen (siehe aber Baltes \& Kliegl, 1992). Angesichts der kumulierten Lebenserfahrung älterer Menschen handelt es sich bei diesen wenigen Stunden im Labor um eine eher zu vernachlässigende Größe. Da viele ältere Personen großes Interesse an einem kognitiv und körperlich aktiven Lebensstil haben, erscheint es nicht nur wünschenswert, sondern auch praktisch möglich, zukünftige Interventionsstudien stärker in den Alltag zu integrieren und das Ausmaß des Trainings, und damit womöglich dessen physiologische Auswirkungen auf das Gehirn, um einige Größenordnungen zu steigern. So wird am Max-Planck-Institut für Bildungsforschung gegenwärtig eine Interventionsstudie in den Bereichen Arbeitsgedächtnis, episodisches Gedächtnis und Wahrnehmungsgeschwindigkeit durchgeführt, bei der 100 junge und 100 ältere Erwachsene über einen Zeitraum von über 100 Sitzungen trainiert werden (siehe auch Lindenberger et al., 2007). Zusätzlich zu der Erfassung des Verhaltens, die neben kognitiven und körperlichen auch motivationale und persönlichkeitsbezogene Bereiche abdecken sollte, sollten in umfangreichen Interventionsstudien dieser Art zu Beginn, am Ende und zu späteren Zeitpunkten auch neurostrukturelle und neurofunktionale Maße erfasst werden, um Altersunterschiede im Ausmaß und Verhältnis der Plastizität von Verhalten und Gehirn zu erhellen und zu individuell abgestimmten Empfehlungen für geeignete Interventionen zu gelangen.

\section{Optimales, normales und pathologisches kognitives Altern}

Die an der normalen kognitiven Alterung beteiligten Prozesse sind den Prozessen, die am Auftreten dementieller Erkrankungen beteiligt sind, oft erstaunlich ähnlich; eine klare Trennungslinie lässt sich insbesondere im hohen Alter nicht ziehen. Deswegen bedürfen Forschungsergebnisse zum normalen Altern und zum pathologischen kognitiven Altern einer vergleichenden Betrachtung und Deutung. So zeigen jüngste Forschungsergebnisse, dass die aktive Teilhabe am sozialen Leben den alterungsbedingten Rückgang kognitiver Leistungen im Normalbereich abzuschwächen vermag (Lövdén et al., 2005). Zugleich gibt es Hinweise darauf, dass soziale Teilhabe auch das Auftreten einer Demenz hinauszögern kann (Fratiglioni, Paillard-Borg \& Winblad, 2004). Die Forschung darf sich nicht ausschließlich darauf konzentrieren, Wege zur Vermeidung besonders negativer kognitiver Alterungsverläufe zu finden, denn dies könnte dazu führen, dass Mechanismen, die besonders positive Verläufe ermöglichen, übersehen werden. Ähnlichkeiten und Unterschiede der Mechanismen im unteren und oberen Leistungsbereich sind gleichermaßen von Belang.

\section{Die Berücksichtigung persönlicher Wissensbestände bei der Gestaltung flexibel unterstützender Umwelten}

Um den Nutzen der kognitiven Alternsforschung für die Lebenspraxis älterer Menschen zu steigern, ist es erforderlich, die persönlich geprägten, jeweils unterschiedli- 
chen Wissensbestände älterer Personen stärker zu berücksichtigen. Kognitive Fähigkeiten altern nicht einheitlich; die biologische Alterung steht in einem dynamischen Austausch mit den Früchten des lebenslangen Lernens. Immer dann, wenn biographisch erworbene Wissensbestände im Vordergrund stehen und entscheidend zu kognitiven Leistungen beitragen, können Personen mit zunehmendem Alter gleichbleibend hohe oder sogar ansteigende Leistungen zeigen. Diese Wissensbestände sind nicht immer leicht zu erfassen, und sie lassen sich nur eingeschränkt zwischen verschiedenen Personen vergleichen, da sie dem besonderen Erfahrungshintergrund jeder einzelnen Person entsprechen (Staudinger \& Pasupathi, 2000).

Die Berücksichtigung persönlichen Wissens, zu dem auch implizite (unbewusste) Angewohnheiten und Vorlieben gehören, können zum Beispiel dem Einsatz von Technologie im Alter neue Perspektiven eröffnen (vgl. Lindenberger, 2007a). Gegenwärtig entsteht bisweilen der Eindruck, ältere Menschen sollten sich an die Erfordernisse der Technik anpassen. Häufig jedoch ist das Gegenteil sinnvoll und mittlerweile auch technisch möglich. Demnach könnten Ingenieure und Psychologen ältere Personen als „Experten ihres eigenen Lebens“ begreifen, die ein reichhaltiges Wissen über ihre persönlichen Vorlieben, Gewohnheiten und Besonderheiten besitzen. Zugleich fällt es älteren Personen bisweilen schwerer, ihr Wissen an Ort und Stelle einzusetzen - etwa wenn sie müde sind, sie abgelenkt werden oder mehrere Ziele gleichzeitig verfolgt werden sollen oder wenn ihre Sinne und ihr Körper die kognitiven Ressourcen auf sich ziehen (s. nächster Abschnitt). Maßgeschneiderte technische Hilfen können in solchen Fällen äußere Hinweisreize (cues) anbieten, die ältere Erwachsene darin unterstützen, ihre Ziele nicht aus den Augen zu verlieren und beabsichtigte Handlungen auch tatsächlich durchzuführen. Voraussetzung für eine solche, flexibel und individuell unterstützende Technologie wäre, dass die Technik zunächst die Gewohnheiten und Vorlieben ihrer Nutzer erlernt.

\section{Das Altern von Sensomotorik und Kognition: Ein Überblick}

Wie bereits weiter oben gefordert wurde, bedürfen die altersbezogenen Veränderungen in verschiedenen Funktionsbereichen der gemeinsamen Betrachtung und Erforschung, um zu einem systemischen (ganzheitlichen) Verständnis des Alterns zu gelangen. Dies soll abschließend an einem weiteren Gegenstand der aktuellen Forschung, dem Verhältnis zwischen Kognition und Sensomotorik im Alter, beispielhaft dargestellt werden. Ausgangspunkt der Überlegungen zum Verhältnis zwischen Sensomotorik und Kognition im Alter ist der Gedanke, dass der Aufmerksamkeitsbedarf sensomotorischer Anforderungen im Laufe des Erwachsenenalters deutlich zunimmt. Man denke zum Beispiel daran, wieviel Aufmerksamkeit es einen 20-Jährigen und einen 85-Jährigen kostet, eine belebte Autostraße als Fußgänger zu überqueren, womöglich bei tiefstehender Sonne im Gegenlicht. „Körper“ ist also mit fortschreitendem Alter immer mehr auf „Geist“" angewiesen, dessen relevante Aspekte selbst wiederum von der Alterung betroffen sind. Im Folgenden sei summarisch dargestellt, wie kognitive, sensorische und sensomotorische Funktionen im Laufe des Erwachsenenalters nach- 
lassen und wie diese Leistungseinbußen miteinander in Wechselwirkung stehen (s. a. Schaefer et al., 2006).

\section{Kognition}

Das Verhalten von Menschen steht nicht unter der direkten Kontrolle von Sinnesreizen, sondern wird, mehr als bei jedem anderen Lebewesen, durch interne Repräsentationen von Handlungszielen und Handlungsmitteln bestimmt. Deswegen erfolgt die Regulation von Wahrnehmen, Handeln und Denken zu einem großen Teil „,top down“ statt „,bottom up“ und erfordert bewusste geistige Anstrengung oder kontrollierte Aufmerksamkeit (vgl. Lövdén \& Lindenberger, 2007). Die Auswirkungen alterungsbedingter Einbußen in der kontrollierten Aufmerksamkeit oder der Fähigkeit, gemäß unseren Intentionen und Plänen zu handeln, treten je nach Aufgabe und Kontext mehr oder minder deutlich zutage. Wenn Aufgaben klar strukturiert sind und ablenkende Reize fehlen, so sind sowohl die Kontrollanforderungen als auch die Alterseinbußen gering. Wenn hingegen mehrere Aufgaben gleichzeitig bearbeitet werden oder wenn Handlungsziele mit der Wahrnehmung in Konflikt geraten, dann sind die Kontrollanforderungen hoch, und die alterungsbedingten Einbußen ebenfalls.

Das Arbeitsgedächtnis dient der kontrollierten Aufmerksamkeit und bezeichnet die Fähigkeit, Informationen in der Aufmerksamkeit aktiv zu halten und sie gleichzeitig zu bearbeiten. Im Arbeitsgedächtnis werden Handlungsziele verändert und koordiniert, wenn mehrere oder komplexe Aufgaben bearbeitet werden. Auch bei Aufgaben, die das Arbeitsgedächtnis belasten, sind deutliche Leistungseinbußen die Norm.

Schließlich ist die Assoziationsbildung (binding) im Alter beeinträchtigt; der Ort, die Zeit und der Inhalt von Ereignissen werden weniger zuverlässig aneinander gebunden als im Kindes- und Erwachsenenalter. Außerdem greifen die Bindungsprozesse beim Wahrnehmen, Einprägen und Erinnern nicht so gut ineinander, so dass die Repräsentationen verschiedener Ereignisse weniger gut voneinander unterschieden werden können. Neue Assoziationen werden weniger leicht gebildet und gefestigt, und bereits vorhandene Assoziationen werden weniger leicht abgerufen. Gedächtnis und Lernen sind von diesen Einbußen insbesondere dann betroffen, (a) wenn die Inhalte neu und assoziativ komplex sind, (b) wenn sie der Gewohnheit und dem bereits vorhandenen Wissen zuwiderlaufen und (c) wenn die Umwelt keine Hinweise bietet, die das Einprägen oder Erinnern erleichtern.

\section{Sehen}

Wie alle anderen Sinnesleistungen lässt der Sehsinn mit dem Alter nach. Bereits im mittleren Erwachsenenalter nimmt die Anpassung der Sehschärfe im Nahbereich ab (Nagel, Werkle-Bergner, Li \& Lindenberger, 2007). Auch die Sehschärfe, die Kontrastwahrnehmung und das Farbensehen verändern sich. Später kommen die Zunahme der Blendempfindlichkeit sowie Schwierigkeiten bei der Anpassung an Helligkeitsunterschiede hinzu. Ab etwa 60 Jahren lässt sich bei den meisten Personen eine Abnahme des Sehfeldes nachweisen. Im Vergleich zu jungen Erwachsenen müssen Reize länger, mit mehr Kontrast und näher am Zentrum des Sehfeldes dargeboten werden, um wahrgenommen zu werden. 


\section{Hören}

Nach den Kriterien der Weltgesundheitsorganisation lässt sich bei $20 \%$ der 40 - bis 50-jährigen Erwachsenen eine Hörbeeinträchtigung nachweisen. Bei den 70- bis 80-Jährigen steigt dieser Anteil auf 75\%. Insbesondere hohe Töne werden weniger gut wahrgenommen. Schwierigkeiten im Verständnis gesprochener Sprache sind die wichtigste Folge alterungsbedingter Höreinbußen. Die meisten Personen über 80 Jahre verstehen etwa $25 \%$ der Wörter einer Unterhaltung nicht richtig. Durch die gleichzeitige Abnahme der kontrollierten Aufmerksamkeit fällt es vielen älteren Erwachsenen schwer, sich an Unterhaltungen in lauten Umgebungen wie Restaurants und Bars zu beteiligen. Das Sprachverständnis leidet besonders dann, wenn die Umgebung laut ist, wenn schnell gesprochen wird, wenn mehrere Personen am Gespräch teilnehmen oder wenn der Gegenstand des Gesprächs komplex und neu ist. Wie auch beim Sehsinn lassen sich Einbußen in Hörleistungen am besten als Wechselwirkung zwischen sensorischen Veränderungen wie dem Verlust von Haarzellen im Innenohr und kognitiven Veränderungen wie dem nachlassenden Arbeitsgedächtnis begreifen.

\section{Gleichgewichtskontrolle}

Die Gleichgewichtskontrolle baut auf vielen Sinnesleistungen auf; sein Gleichgewicht $\mathrm{zu}$ halten erfordert das koordinierte Zusammenspiel visueller, auditorischer, vestibulärer und propriozeptiver Sinnesleistungen. Alle diese Sinne nehmen im Alter ab. Der Alterungsprozess führt zu weniger zuverlässigen sensorischen Informationen, einer ungenaueren Integration dieser Informationen und zu weniger effizienten Ausgleichsbewegungen zum Erhalt des Gleichgewichts. Insbesondere das Gehen ist von diesen Veränderungen betroffen. Die Zunahme von Stürzen im Alter ist das folgenreichste Anzeichen für alterungsbedingte Schwierigkeiten in der Gleichgewichtskontrolle.

\section{Das Zusammenwirken von Kognition, Sensorik und Sensomotorik im Alter}

Wie bereits in den vergangenen Abschnitten deutlich wurde, verändert sich auch das Zusammenwirken von Kognition, Sensorik und Sensomotorik im Laufe des Erwachsenenalters. Junge Erwachsene müssen nur einen geringen Anteil ihrer kognitiven Ressourcen in das Sehen, das Hören oder die Gleichgewichtskontrolle investieren, da diese sensorischen und sensomotorischen Funktionen weitgehend automatisch reguliert werden. Im Alter ändert sich dieses Bild: Sehen, Hören und Gleichgewichtskontrolle sind zunehmend auf den Einsatz kognitiver Ressourcen angewiesen. Leider nehmen aber genau jene kognitiven Ressourcen, die dazu besonders vonnöten sind, nämlich die kontrollierte Aufmerksamkeit, das Arbeitsgedächtnis und die Assoziationsbildung, ebenfalls besonders deutlich mit dem Alter ab. Der biologische Alterungsprozess führt also in ein Dilemma, weil zunehmend benötigte Ressourcen selbst im Abnehmen begriffen sind (Lindenberger, Marsiske \& Baltes, 2000). Ein Hauptzweck des Einsatzes von Technologie im Alter könnte darin bestehen, diesem Dilemma die Spitze zu nehmen. 
Die Ergebnisse einer altersvergleichenden experimentellen Studie sollen exemplarisch verdeutlichen, dass das Gehen mit zunehmendem Alter die Aufmerksamkeit tatsächlich immer stärker beansprucht (Lindenberger et al., 2000). Die jungen und älteren Erwachsenen, die an dieser Studie teilnahmen, wurden zunächst im Sitzen in einer Gedächtnistechnik instruiert und trainiert. Am Ende des Trainings waren alle Erwachsene, die älteren ebenso wie die jungen, in der Lage, sich im Durchschnitt an je 10-12 von insgesamt 16 Wörtern einer Wortliste in der richtigen Reihenfolge zu erinnern. Nach dem Training wurden die Bedingungen variiert, in denen sich die Probanden die Wortlisten einprägen sollten - im Sitzen, im Stehen, beim Laufen einer einfachen Wegstrecke oder beim Laufen einer komplexen Wegstrecke.

Sowohl die negativen Auswirkungen des gleichzeitigen Gehens auf die Gedächtnisleistung als auch die negativen Auswirkungen des gleichzeitigen Einprägens von Wörtern auf die Schnelligkeit und Genauigkeit des Gehens nahmen mit dem Alter zu. Bei der Gedächtnisleistung war die Zunahme dieser sogenannten Doppelaufgabenkosten besonders ausgeprägt (Abb. 3). Hier zeigten bereits Personen im mittleren Erwachsenenalter größere Leistungseinbußen als junge Erwachsene. Außerdem waren die Kosten bei der komplexen Wegstrecke generell größer als die Kosten bei der einfachen Wegstrecke - ein weiterer Hinweis dafür, dass die Leistungseinbußen im Gedächtnisbereich mit einem Mehrbedarf an kontrollierter Aufmerksamkeit für das Gehen einhergehen. Offensichtlich beanspruchte das Gehen bei den älteren Probanden einen größeren Anteil an kognitiven Ressourcen als bei den jüngeren, die dann beim Bearbeiten der Gedächtnisaufgabe fehlten.

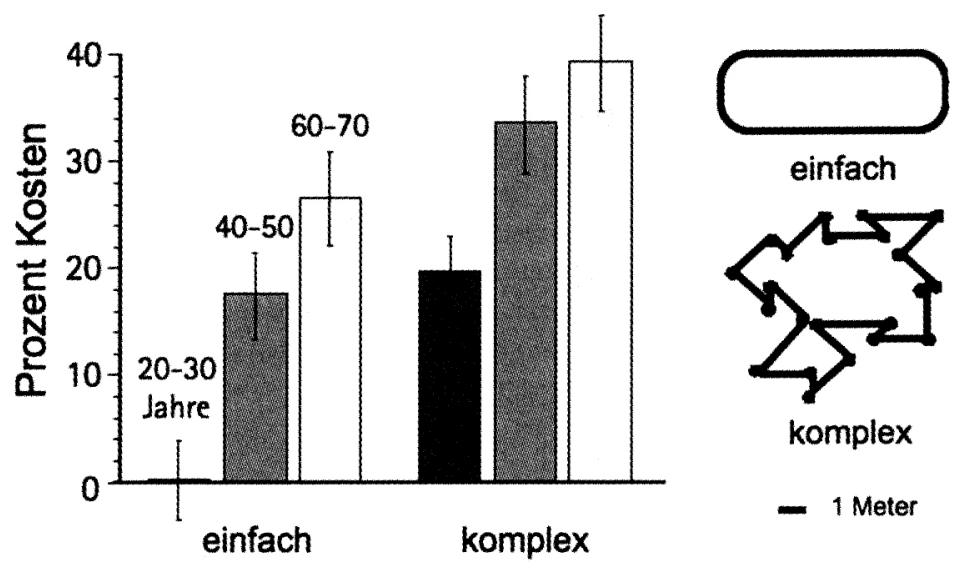

Abb. 3. Der Aufmerksamkeitsbedarf des Gehens nimmt im Laufe des Erwachsenenalters zu.

Dargestellt sind die Auswirkungen des Gehens auf die Gedächtnisleistung. Die gezeigten Doppelaufgabenkosten beziehen sich auf die Abnahme der Gedächtnisleistung beim Gehen auf einer ovalen und einer unregelmäßigen Wegstrecke im Vergleich zur Gedächtnisleistung beim Sitzen und Stehen. Personen im mittleren und höheren Erwachsenenalter zeigen deutlich größere Doppelaufgabenkosten als junge Erwachsene. Der Grundriss der beiden Wegstrecken ist unter dem Säulendiagramm aufgetragen. Modifiziert nach Lindenberger, Marsiske und Baltes (2000). 


\section{Ausblick}

Die Erforschung der Plastizität menschlichen Verhaltens gestattet Einblicke in die menschliche Entwicklung, die sowohl aus grundlagenwissenschaftlicher als auch aus gesellschaftlicher Perspektive von besonderem Interesse sind. Um hinsichtlich Ursachen und Bandbreite von Entwicklungsverläufen zu einem besseren Verständnis $\mathrm{zu}$ gelangen, ist die Bestimmung neuronaler und psychischer Mechanismen, die „Veränderungen in Veränderungen“ ermöglichen, mit ihnen einhergehen und ihnen folgen, zentral. Aus diesem Grund wird ein Schwerpunkt der künftigen kognitiven Alternsforschung in der Verknüpfung von kognitiven Trainingsprogrammen und längsschnittlichen Beobachtungen (vgl. Schaie \& Willis, 1986; Singer et al., 2003) unter Einbezug neurochemischer, neurofunktionaler und neuroanatomischer Maße liegen (Lindenberger et al., 2007).

Aus angewandter Sicht wird die Frage forschungsleitend sein, welche Konstellationen von Verhaltensweisen und Lebensstilen das Überhandnehmen chronischer kognitiver Einschränkungen, die eine selbständige Lebensführung gefährden und schließlich unmöglich machen, möglichst weit hinausschieben. Die Fragen „Was ist Altern?" und „Was könnte Altern sein?" sind in diesem Sinne untrennbar miteinander verknüpft (vgl. Baltes, 1987; Lindenberger, 2007b; Tetens, 1777).

\section{Literatur}

Allaire, J. C. \& Marsiske, M. (2005). Intraindividual variability may not always indicate vulnerability in elders' cognitive performance. Psychology and Aging, 20, 390-401.

Bäckman, L., Nyberg, L., Lindenberger, U., Li, S.-C. \& Farde, L. (2006). The correlative triad among aging, dopamine, and cognition: Current status and future projects. Neuroscience and Biobehavioral Reviews, 30, 791-807.

Baltes, P. B. (1987). Theoretical propositions of life-span developmental psychology: On the dynamics between growth and decline. Developmental Psychology, 23, 611-626.

Baltes, P. B. \& Kliegl, R. (1992). Further testing of limits of cognitive plasticity: Negative age differences in a mnemonic skill are robust. Developmental Psychology, 28, 121-125.

Baltes, P. B., Lindenberger, U. \& Staudinger, U. M. (2006). Life span theory in developmental psychology. In W. Damon \& R. M. Lerner (Eds.), Handbook of child psychology: Vol. 1. Theoretical models of human development (6th ed., pp. 569-664). New York: Wiley.

Baltes, P. B., Reuter-Lorenz, P.A. \& Rösler, F. (Eds.) (2006). Lifespan development and the brain: The perspective of biocultural co-constructivism. New York: Cambridge University Press.

Brehmer, Y., Li, S.-C., Müller, V., Oertzen, T. von \& Lindenberger, U. (2007). Memory plasticity across the lifespan: Uncovering children's latent potential. Developmental Psychology, 43, $465-478$.

Brehmer, Y. \& Lindenberger, U. (2008). Kognitive Leistungsreserven im höheren Erwachsenenalter: Befunde der Interventionsforschung. In F. Petermann \& W. Schneider (Hrsg.) Enzyklopädie der Psychologie: C, V, Bd. 7. Angewandte Entwicklungspsychologie (S. 917 947). Göttingen: Hogrefe.

Dittmann-Kohli, F., Lachman, M.E., Kliegl, R. \& Baltes, P. B. (1991). Effects of cognitive training and testing on intellectual efficacy beliefs in elderly adults. Journal of Gerontology: Psychological Sciences, 46, P162-P164. 
Fratiglioni, L., Paillard-Borg, S. \& Winblad, B. (2004). An active and socially integrated lifestyle in late life might protect against dementia. Lancet Neurology, 3, 343-353.

Ghisletta, P. \& Lindenberger, U. (2003). Age-based structural dynamics between perceptual speed and knowledge in the Berlin Aging Study: Direct evidence for ability dedifferentiation in old age. Psychology and Aging, 18, 696-713.

Ghisletta, P. \& Lindenberger, U. (2004). Static and dynamic longitudinal structural analyses of cognitive changes in old age. Gerontology, 50, 12-16.

Kempermann, G. (2005). Adult neurogenesis: Stem cells and neuronal development in the adult brain. Oxford, UK: Oxford University Press.

Li, S.-C. (2003). Biocultural orchestration of developmental plasticity across levels: The interplay of biology and culture in shaping the mind and behavior across the life span. Psychological Bulletin, 129, 171-194.

Li, S.-C., Huxhold, O. \& Schmiedek, F. (2004). Aging and processing robustness: Evidence from cognitive and sensorimotor functioning. Gerontology, 50, 28-34.

Li, S.-C. \& Lindenberger, U. (1999). Cross-level unification: A computational exploration of the link between deterioration of neurotransmitter systems and dedifferentiation of cognitive abilities in old age. In L.-G. Nilsson \& H. J. Markowitsch (Eds.), Cognitive neuroscience of memory (pp. 103-146). Seattle, WA: Hogrefe \& Huber.

Li, S.-C. \& Lindenberger, U. (2002). Co-constructed functionality instead of functional normality [Invited commentary]. Behavioral and Brain Sciences, 25, 761-762.

Li, S.-C., Lindenberger, U. \& Sikström, S. (2001). Aging cognition: From neuromodulation to representation. Trends in Cognitive Sciences, 5, 479-486.

Li, S.-C., Oertzen, T. von \& Lindenberger, U. (2006). A neurocomputational model of stochastic resonance and aging. Neurocomputing, 69, 1553-1560.

Lindenberger, U. (2007a). Technologie im Alter: Chancen aus Sicht der Verhaltenswissenschaften. In P. Gruss (Hrsg.), Die Zukunft des Alterns: Die Antwort der Wissenschaft (S. 221-239). München: C. H. Beck.

Lindenberger, U. (2007b). Historische Grundlagen: Johann Nicolaus Tetens als Wegbereiter des Lebensspannen-Ansatzes in der Entwicklungspsychologie. In J. Brandtstädter \& U. Lindenberger (Hrsg.), Entwicklungspsychologie der Lebensspanne: Ein Lehrbuch (S. 933). Stuttgart: Kohlhammer.

Lindenberger, U., Li, S.-C. \& Bäckman, L. (Eds.) (2006). Methodological and conceptual advances in the study of brain-behavior dynamics: A multivariate lifespan perspective [Special issue]. Neuroscience and Biobehavioral Reviews, 30 (6).

Lindenberger, U., Li, S.-C., Lövdén, M. \& Schmiedek, F. (2007). The Center for Lifespan Psychology at the Max Planck Institute for Human Development: Overview of conceptual agenda and illustration of research activities. International Journal of Psychology, 42, 229242.

Lindenberger, U., Marsiske, M. \& Baltes, P. B. (2000). Memorizing while walking: Increase in dual-task costs from young adulthood to old age. Psychology and Aging, 15, 417-436.

Lövdén, M., Ghisletta, P. \& Lindenberger, U. (2005). Social participation attentuates cognitive decline in perceptual speed in old and very old age. Psychology and Aging, 20, 423-434.

Lövdén, M., Li, S.-C., Shing, Y.L. \& Lindenberger, U. (2007). Within-person trial-to-trial variability precedes and predicts cognitive decline in old and very old age: Longitudinal data from the Berlin Aging Study. Neuropsychologia, 45, 2827-2838.

Lövdén, M. \& Lindenberger, U. (2007). Intelligence. In J. E. Birren (Ed.), Encyclopedia of gerontology: Age, aging, and the aged (2nd ed., Vol. 1, pp. 763-770). Amsterdam: Elsevier. 
MacDonald, S. W. S., Hultsch, D. F. \& Dixon, R. A. (2003). Performance variability is related to change in cognition: Evidence from the Victoria Longitudinal Study. Psychology and Aging, 18, 510-523.

McArdle, J. J. \& Hamagami, F. (2001). Latent difference score structural models for linear dynamic analyses with incomplete longitudinal data. In L. M. Collins \& A. G. Sayer (Eds.), New methods for the analysis of change (pp. 137-176). Washington, DC: American Psychological Association.

Molenaar, P.C. M. (in press). The nonequivalence of structures of inter- and intra-individual variation associated with nonergodic psychological processes. Current Directions in Psychological Science.

Molenaar, P. C. M., Boomsma, D. I. \& Dolan, C.V. (1991). Genetic and environmental factors in a developmental perspective. In D. Magnusson, L. R. Bergman, G. Rudinger \& B. Törestad (Eds.), Problems and methods in longitudinal research: Stability and change (pp. 250-273). Cambridge, UK: Cambridge University Press.

Nagel, I. E., Werkle-Bergner, M., Li, S.-C. \& Lindenberger, U. (2007). Perception. In J.E. Birren (Ed.), Encyclopedia of gerontology: Age, aging, and the aged (2nd ed., Vol. 2, pp. 334-342). Amsterdam: Elsevier.

Nesselroade, J. R., Gerstorf, D., Hardy, S. A. \& Ram, N. (2007). Idiographic filters for psychological constructs. Measurement: Interdisciplinary Research and Perspectives, 5, 217-235.

Schaefer, S., Huxhold, O. \& Lindenberger, U. (2006). Healthy mind in healthy body? A review of sensorimotor-cognitive interdependencies in old age. European Review of Aging and Physical Activity, 3, 45-54.

Schaie, K.W. \& Willis, S. L. (1986). Can adult intellectual decline be reversed? Developmental Psychology, 22, 223-232.

Schmiedek, F. \& Lindenberger, U. (2007). Methodologische Grundlagen. In J. Brandtstädter \& U. Lindenberger (Hrsg.), Entwicklungspsychologie der Lebensspanne: Ein Lehrbuch (S. 67-96). Stuttgart: Kohlhammer.

Siegler, R. S. (1994). Cognitive variability: A key to understanding cognitive development. Current Directions in Psychological Science, 3, 1-5.

Singer, T., Lindenberger, U. \& Baltes, P. B. (2003). Plasticity of memory for new learning in very old age: A story of major loss? Psychology and Aging, 18, 306-317.

Staudinger, U. M. \& Pasupathi, M. (2000). Life-span perspectives on self, personality and social cognition. In F. I. M. Craik \& T. A. Salthouse (Eds.), The handbook of aging and cognition (pp. 633-688). Mahwah, NJ: Erlbaum.

Tetens, J. N. (1777). Philosophische Versuche über die menschliche Natur und ihre Entwicklung. Leipzig: Weismanns Erben und Reich (Nachdruck der Kantgesellschaft 1913, Bd. 1. Berlin: Reuther und Reichard).

Wohlwill, J. F. (1970). Methodology and research strategy in the study of developmental change. In L. R. Goulet \& P. B. Baltes (Eds.), Life-span developmental psychology: Research and theory (pp. 150-193). New York: Academic Press. 